ISSN: 2602-8506

Vol. 3, N4., p.44-66, octubre - diciembre, 2019

Recibido: 04-08-2019/Aceptado:07-09-2019/ Publicado: 05-10-2019

\title{
Desafíos de la responsabilidad social corporativa (RSC) en el sector económico popular y solidario del Ecuador
}

\section{Challenges of corporate social responsibility (CSR) in the popular and solidarity economic sector of Ecuador}

Juan Carlos Muyulema Allaica. ${ }^{1}$, Paola Martina Pucha Medina. ${ }^{2}$, Andrea Elizabeth Villamarín Arévalo. ${ }^{3} \&$ Carina Alexandra Muyulema Allaica. ${ }^{4}$

DOI: https://doi.org/10.33262/visionariodigital.v3i4.965

\begin{abstract}
This article aims to identify the challenges in the development of the popular and solidarity economic sector (PSES) of Ecuador, with respect to corporate social responsibility (CSR). To achieve the objective, a retrospective vision of the subject framed in the research line was made to know the different limitations in terms of its evolution and terminology, the approaches and dimensions, measurement tools and different results in organizations that have applied CSR. Then, the origin, legal basis, structure and evolution of the Ecuadorian PSES were externalized. Finally, the discussion on the current challenges of the PSES against the CSR, regarding the terms of the three dimensions of sustainability must be in balance: economic, environmental and social. The analyzed results affected analogies between the dimensions of CSR with the principles of the PSES, part of the conception of its conception, specifying that the CSR will contribute through the efficient management of a survival capacity of the sector. In this sense, the advantages of implementing CSR to PSES organizations are considerable for economic recovery, due to their contributions in the progress of knowledge, combining the procedures and values of the Popular and Solidarity Economy (PSE), by incorporating the basis of the triple line of performance with those of the emerging sectors, contributing to security for a good life.
\end{abstract}

1 Pontificia Universidad Católica del Ecuador Sede Ambato (PUCESA), Escuela de Diseño Industrial. Ambato, Ecuador., juanca327@hotmail.com

${ }^{2}$ Universidad de Cantabria, Santander, Facultad de Ciencias Económicas y Empresariales. Santander, España., pumepm@hotmail.com

3 Universidad Estatal de Bolívar, Facultad de Ciencias de Salud y del Ser Humano. Guaranda, Ecuador., aeva85@hotmail.com

4 Grupo Consultor Empresarial CAAPTES-Ecuador, Departamento de Gestión de Proyectos de Inversión. Riobamba, Ecuador, karina-muyulema94@ hotmail.com 
ISSN: 2602-8506

Vol. 3, N4., p.44-66, octubre - diciembre, 2019

Keywords: Ecuador, Corporate social responsibility (CSR), Popular and Solidarity Economic Sector (PSES), Sustainability.

\section{Resumen}

A través del presente artículo se pretende identificar los desafíos en el desarrollo del sector económico popular y solidario (SEPS) del Ecuador, con respecto a la responsabilidad social corporativa (RSC). Para lograr el objetivo, se efectuó una visión retrospectiva de la temática enmarcada en la línea de investigación, permitiendo conocer las distintas definiciones en cuanto a su evolución y terminología, los enfoques y dimensiones, herramientas de medición y diferentes resultados en organizaciones que han aplicado RSC. Seguidamente se exteriorizó el origen, base legal, estructura y evolución del SEPS ecuatoriano. Por último, se entabló la discusión sobre los retos actuales del SEPS frente a las RSC, en cuanto a términos de las tres dimensiones de la sostenibilidad que deben estar en equilibrio: económica, medioambiental y social. Los resultados analizados demuestran analogías entre las dimensiones de RSC con los principios del SEPS, considerada parte innata de su concepción, especificando que la RSC aportaría a través de la eficiente gestión una capacidad de supervivencia del sector. En este sentido, las ventajas de implantar la RSC a las organizaciones del SEPS son considerables para la reactivación económica por sus aportaciones en el progreso del conocimiento, combinando los procedimientos y valores propios de la economía popular y solidario (EPS), al incorporar a ello la base de la triple línea de rendimiento con las de los sectores emergentes, coadyuvando a la seguridad para el buen vivir.

Palabras claves: Ecuador, Responsabilidad social corporativa (RSC), Sector económico popular y solidario (SEPS), Sostenibilidad.

\section{Introducción}

El abanico de léxicos enmarcados dentro de economía social, ha traído consigo la responsabilidad social corporativa (RSC) como término imperante dese una perspectiva de desarrollo sostenible, debido a las transformaciones estructurales de las economías. Este término puede comprenderse como la cohesión voluntaria de las organizaciones para con sus partes implicadas, asumiendo triple dimensión; económica, social y ambiental; que conlleve a compromiso más allá de las exigencias reglamentarias permitiendo incorporarse de manera activa y fehaciente en el entorno.

La dimensión de RSC más ampliamente estudiada es, con mucho, la económica, lo que crea consenso entre los estudios. La segunda dimensión más estudiada es el ambiente (Orlitzky \& Shen, 2013; Carro, J., Sarmiento, S. \& Rosano G., 2017). Sin embargo, los factores sociales son mucho menos estudiados (Muyulema, 2018). El meta análisis de Horváthová (2010) de estudios ecológicos advierte que los coeficientes de correlación simples generan resultados más 
negativos al vincular el desempeño con factores ecológicos, así como, también señalan la importancia de una cobertura de tiempo apropiada para establecer un vínculo positivo entre el desempeño ambiental y financiero.

La revisión de la literatura relacionada con las relaciones entre la RSC y el sector económico popular y solidario (SEPS), ha sido ampliamente discutida en investigaciones recientes en referencia a problemas con conflictos entre varios intereses de los interesados (Saltos, Mayorga, \& Ruso, 2016; Chávez, R. \& Monzón, J. (2018); Sahut, Peris, \& Teulon, 2019). Una gran parte de la literatura defiende la idea de que la adopción de políticas de RSC conduce a la implementación de nuevas normas normativas y mejores mecanismos del SEPS dentro de una sociedad (Arteaga R., 2017). Los resultados de estudios previos siguen sin ser concluyentes y, como mínimo, requieren más investigación.

La Constitución de la República del Ecuador (2008a), en su artículo 283 define al sistema económico como social y solidario, y a su vez, reconoce diversas formas de organización económica: pública, privada, mixta, y popular y solidaria. Adicionalmente, la publicación de la Ley Orgánica de Economía Popular y Solidaria (LOEPS, 2011) y su reforma en el 2018, incorporó un adelanto hacia el reconocimiento y fortalecimiento del sector, puesto que, a partir de este instrumento, se generó una nueva institucionalidad encaminada al fomento y promoción; estabilidad, solidez y educado funcionamiento de los actores que conforman el mencionado sector. Es así que, la creación de la Superintendencia de Economía Popular y Solidaria en junio de 2012 constituye un adelanto significativo en la cimentación de una arquitectura institucional pública organizada a la rectoría, regulación, control y supervisión del SEPS. Los objetivos de esta ley se fundamentan en reconocer, estimular y fortalecer de forma enérgica el sector que genera empleo e ingresos, pero que en ningún tiempo se habían tenido en cuenta hasta ahora. Además, la ley instaura un marco legal para aquellos que la componen; las cooperativas, las asociaciones y la comunidad. Del mismo modo, define un sistema de derechos, obligaciones y beneficios para los individuos o las organizaciones e implementa las instituciones públicas que adquieren la tarea de regulación, supervisión, promoción y apoyo al bienestar y el bien común.

En Ecuador, la SEPS se define como el conjunto de formas de organización económica y social en las que sus integrantes, con un sustantivo colectivo o individual, despliegan procesos de producción, intercambio, mercantilización, financiación y consumo de bienes y servicios. Las formas de organización del SEPS encierran a las organizaciones de la Economía Popular y Solidaria (EPS) y del Sector Financiero Popular y Solidario (SFPS) (Pucha, Muyulema, Burgos, \& Buenaño, 2019).

Este sector se caracteriza por una pluralidad de actores que, de acuerdo con el marco normativo institucional actual, conforman los sectores cooperativo, asociativo y comunitario (Saltos, Mayorga, \& Ruso, 2016). Estas organizaciones realizan sus actividades basadas, a priori, en relaciones de solidaridad, cooperación y reciprocidad, y ubican al ser humano como sujeto y fin 
ISSN: 2602-8506

Vol. 3, $\mathrm{N}^{\circ} 4 .$, p.44-66, octubre - diciembre, 2019

de toda actividad económica por sobre el lucro, la competencia y la acumulación de capital (Pucha, et al., 2019). De manera más específica y para efectos de este documento, las organizaciones de la Economía Popular y Solidaria se clasifican en: i) cooperativas de producción; ii) cooperativas de consumo; iii) cooperativas de vivienda; iv) cooperativas de servicios; v) asociaciones productivas; y, vi) organizaciones comunitarias.

Por su parte, el SFPS está conformado por: i) cooperativas de ahorro y crédito; ii) cajas solidarias y de ahorro; iii) cajas centrales; y iv) bancos comunales. Estas organizaciones se identifican, en principio, por su lógica asociativa y realizan actividades de intermediación financiera y de responsabilidad social con sus socios, situando a las finanzas al servicio de las necesidades de las personas (Saltos, Mayorga, \& Ruso, 2016; Pucha et al., 2019).

Adicionalmente, el sector está integrado por otro tipo de formas económicas conocidas como Unidades Económicas Populares (UEP), las cuales incluyen actores como: emprendimientos unipersonales, familiares, domésticos, comerciantes minoristas, talleres artesanales y personas responsables de la economía del cuidado (trabajo no remunerado) (Vega, 2016). Este último tipo de economía, aunque con críticas apuntan al trabajo no remunerado que se realiza en el hogar, concerniente con mantenimiento de la vivienda, los cuidados a otras personas del hogar o la comunidad y el mantenimiento de la fuerza de trabajo remunerado.

Este trabajo tiene como objetivo identificar los desafíos del SEPS con respecto a la RSC, buscar la reciprocidad entre la RSC y el SEPS ecuatoriano, detectando los principios enmarcados y el aporte que estas brindan a las partes implicadas y su entorno, a fin de disponer de un desarrollo sostenible de sus partes.

Para dar cumplimiento al objetivo propuesto se han revisado gran cantidad de trabajos relacionados con la temática, estructurando el documento en tres epígrafes. En el primero, se efectúa la revisión literaria del término RSC, examinando la evolución de las distintas definiciones al igual que su terminología, los enfoques y dimensiones, herramientas de medición que se aplican y las diferentes organizaciones que han instaurado la RSC a nivel mundial. El segundo epígrafe se centra en dar a conocer al SEPS del Ecuador, su origen, base legal, estructura y evolución y en el tercer epígrafe; se detiene a discutir los retos actuales del SEPS frente a las RSC, partiendo de la relación entre la RSC y SEPS ecuatoriano, en cuanto a términos de sostenibilidad, económica, social y medioambiental. Culminando en conclusiones para posteriormente presentar la bibliografía utilizada.

\section{Metodología}

Para dar cumplimiento al objeto de estudio, que se centró en identificar los desafíos del SEPS con respecto a la RSC, buscar la reciprocidad entre la RSC y el SEPS ecuatoriano, detectando los principios enmarcados y el aporte que estas brindan a las partes implicadas y su entorno, a 
fin de disponer de un desarrollo sostenible de sus partes. La revisión se centró en tres etapas: búsqueda y detección; obtención; y consulta a fin de analizar y conocer la sinergia existente entre RSC y SEPS en el Ecuador. La investigación abordo tres epígrafes, con temas en esencia importantes, donde se perpetró en: i) efectuar una revisión exhaustiva y sistémica de la literatura sobre las iniciativas de la RSC, ii) conocer al SEPS del Ecuador, su origen, base legal, estructura y evolución, proporcionando así, el marco de referencia a partir del cual se formuló el tercer epígrafe; iii) discutir los retos actuales del SEPS frente a las RSC, partiendo de la relación entre la RSC y SEPS ecuatoriano.

En este sentido, la revisión sobre retos y desafíos para del SEPS con respecto a la RSC, al tratarse de una temática multidisciplinar, se podría considerar como un fenómeno singular sobre el cual sería adecuado tener una primera aproximación mediante los casos de estudio, ya que se está en una primera fase exploratoria de la investigación.

Bajo ese contexto, se evidencia la trascendencia del planteamiento del primer epígrafe de investigación que se orientó en efectuar una revisión literaria del término RSC, examinando la evolución de las distintas definiciones al igual que su terminología, los enfoques y dimensiones, herramientas de medición que se aplican y las diferentes organizaciones que han instaurado la RSC a nivel mundial. Este gran volumen de literatura disponible requirió por una parte conocer y discernir la información relevante de la que no lo es, y posteriormente evaluar, juzgar y localizar la documentación.

El segundo epígrafe, se centró en dar a conocer al SEPS del Ecuador, su origen, base legal, estructura y evolución, mediante una revisión exhaustiva y sistémica de la literatura que partió de estudiar los antecedentes del SEPS en el Ecuador, con el fin de entender y comprender caceristas únicas del sector, lo que constituye tipologías sumamente interesantes de investigación.

En el tercer epígrafe, se detuvo a discutir los retos actuales del SEPS frente a las RSC, partiendo de la relación entre la RSC y SEPS ecuatoriano, en cuanto a términos de sostenibilidad, económica, social y medioambiental, que permitió la identificación de la cooperación que incurren el SEPS en la economía para el bienestar del ser humano, puesto que el sector popular y solidario ha venido practicando la RSC desde hace muchos decenios, bajo el título del cooperativismo.

Finalmente, se realizó un conjunto de consideraciones finales relativas a la realización de este trabajo, reflexionando sobre la experiencia que ha supuesto su ejecución y retomado algunos aspectos significativos sobre los resultados obtenidos. Adicionalmente, se presentó las referencias bibliográficas utilizadas, disponiéndolas por orden alfabético de información para identificar las fuentes utilizadas en el trabajo. 


\section{Resultados}

\section{Revisión de la literatura}

\section{a) Conceptos y evolución de RSC}

Las organizaciones han ido evolucionando la manera de sostenerse en la mente del consumidor y el entorno donde se establecieron dentro de la sociedad, esta personalización busca, de una u otra manera, ser parte activa del entorno y ser reconocida en cada uno de los rincones en donde exista vida humana (García, Gómez, Mera, \& Pabón, 2014). Para muchos legendarios de las ciencias empresariales, los factores de RSC; aún no consensuado; conllevan particularidades merecedoras al mundo cambiante, donde las organizaciones deben extender su ideología que permita acoger a los demás grupos de interés que intervienen de manera directa o indirectamente (García \& Madero, 2016; Martínez, González, \& Díaz, 2018; Torres \& Cano, 2019). En la actualidad, la definición de RSC se ha fortalecido, pero discrepando en su terminología; unos más acertados que otros; las diferentes analogías hallados se encuentran: responsabilidad social empresarial, ética empresarial, filantropía, sostenibilidad, triple línea de rendimiento e inversión socialmente responsable (Espinosa, Muyulema, Sánchez, \& Usca, 2019).

La RSC, como fenómeno empresarial inicialmente fue identificada en la década de los 50 por Bowen, expresando en su libro la manera en que las corporaciones deben comportarse con la naturaleza que la rodea desde una perspectiva ética-social. Consiguientemente en la década de los 60 Frederick sostiene que la Responsabilidad Social (RS) destina los recursos existentes a fines sociales, contrastando además que existen tres ideas que prevalecen en este periodo: la del administrador público, equilibrio de la competencia por los recursos corporativos y la filantropía empresarial. A finales de la década de los 70, Carroll define el concepto RSC como aquella responsabilidad que las organizaciones deben considerar en el ámbito económico, legal, ético y filantrópico, quién posteriormente (1991) clasificó tales responsabilidades y creó la "teoría de la pirámide", sirviendo algunas como bases para otras (Espinosa et al., 2019). En la década posterior, se adhiere la "teoría de los Stakeholders"; efectuado por Freeman; donde consideran el apoyo a los diferentes grupos afectados por la empresa. A mediados de la década de los 90 , Drucker señala a la RSC parte relevante de las empresas, la cual se filtra en las diferentes áreas de la organización. En el año 2001 la Comisión Europea genera una definición más amplia en cuanto a la RSC con base a elementos y aportaciones de los diferentes autores, estableciéndola como estrategia indispensable en las organizaciones que cooperan con la sociedad, generando interacción para un desarrollo sostenible en conjunto. En el año 2012, la ISO establece de manera más detallada la definición de la RSC en comparación a la creada por la Unión Europea, agregando a la misma considerar las expectativas de los involucrados, sin embargo, la Organización Internacional del Trabajo (OIT) sintetiza el concepto desde un marco moral que debe ser cumplido por la organización a causa de los recursos extraídos del entorno (Martínez \& Rodríguez, 2013; Chávez, R. \& Monzón, J., 2018). 
ISSN: 2602-8506

Vol. 3, $\mathrm{N}^{\circ} 4 .$, p.44-66, octubre - diciembre, 2019

Bajo estas premisas a continuación en la Tabla 1, se presenta una síntesis de la evolución del concepto de RSC.

Tabla 1. Evolución del concepto de RSC

\begin{tabular}{|c|c|c|}
\hline Autor & País /Año & Concepto de RSC \\
\hline Bowen, $\mathbf{H}$. & $\begin{array}{c}\text { Estados } \\
\text { Unidos/1953 }\end{array}$ & $\begin{array}{l}\text { Las obligaciones de los empresarios para impulsar políticas } \\
\text { corporativas para tomar decisiones o para seguir líneas de } \\
\text { acción que son deseables en términos de los objetivos y } \\
\text { valores de la sociedad. }\end{array}$ \\
\hline Frederick W. & $\begin{array}{c}\text { Estados } \\
\text { Unidos/1960 }\end{array}$ & $\begin{array}{l}\text { La aportación privada a los recursos económicos y humanos } \\
\text { de la sociedad y una voluntad por parte de las empresas para } \\
\text { ver que esos recursos fueron utilizados para fines sociales en } \\
\text { general. }\end{array}$ \\
\hline Carroll Archie & $\begin{array}{l}\text { Estados Unidos } \\
\text { /1999 }\end{array}$ & $\begin{array}{l}\text { La responsabilidad social de las empresas abarca las } \\
\text { expectativas económicas, legales, éticas y discrecionales que } \\
\text { la sociedad tiene de las organizaciones en un momento dado. }\end{array}$ \\
\hline Ducker Peter & Austria/1996 & $\begin{array}{l}\text { Cada organización debe asumir la plena responsabilidad por } \\
\text { el efecto que tenga en sus empleados, en el entorno, en los } \\
\text { clientes y en cualquier persona o cosa que toque. }\end{array}$ \\
\hline Comisión Europea & Brucelas/2001 & $\begin{array}{l}\text { La integración voluntaria, por parte de las empresas, de las } \\
\text { preocupaciones sociales y medioambientales en sus } \\
\text { operaciones comerciales y en sus relaciones con sus } \\
\text { interlocutores. }\end{array}$ \\
\hline $\begin{array}{l}\text { ISO - International } \\
\text { Organization for } \\
\text { Standardization }\end{array}$ & España/2012 & $\begin{array}{l}\text { La Responsabilidad de una organización respecto de los } \\
\text { impactos de sus decisiones y actividades en la sociedad y el } \\
\text { medio ambiente, por medio de un comportamiento } \\
\text { transparente y ético que: contribuya al desarrollo sostenible, } \\
\text { la salud y el bienestar general de la Sociedad; tome en } \\
\text { consideración las expectativas de sus partes interesadas, } \\
\text { stakeholders; esté en cumplimiento con la legislación } \\
\text { aplicable y sea consistente con normas internacionales de } \\
\text { comportamiento; y esté integrada a través de toda la } \\
\text { organización y practicada en sus relaciones. }\end{array}$ \\
\hline $\begin{array}{l}\text { OTI - Organización } \\
\text { Internacional del } \\
\text { Trabajo }\end{array}$ & Ginebra/2010 & $\begin{array}{l}\text { Una iniciativa de carácter voluntario dirigida por las } \\
\text { empresas, rebasando el mero cumplimiento de la ley. }\end{array}$ \\
\hline
\end{tabular}

Fuente: Los autores basados en Martínez \& Rodríguez (2013); Chávez, R. \& Monzón, J. (2018); Espinosa et al., (2019).

\section{b) Dimensiones y enfoques de la RSC}

Parte relevante es conocer la extensión de la RSC en la empresa y su grupo de interés, vista como un constructo multidimensional expresados por diferentes autores. Según Martínez \& Rodríguez (2013) señalan la existencia de varias capacidades en donde se enmarca la RSC como un concepto unidimensional. Sin embargo, lo expuesto por Carroll en 1999, dispuso de mayor hospitalidad con los diferentes autores y su significado; quien sostiene que la longitud de la RSC 
ISSN: 2602-8506

Vol. 3, $\mathrm{N}^{\circ} 4 .$, p.44-66, octubre - diciembre, 2019

es: económica, legal, ética y filantrópica, las cuales se encuentran concatenadas entre sí. Otros puntos de vistas como los autores García \& Madero (2016); Carro, J., Sarmiento, S. \& Rosano G., (2017); Chávez, R. \& Monzón, J. (2018); Espinosa et al. (2019), exteriorizan la medición de la RSC en aspectos económicos, medioambientales y sociales bajo el concepto de desarrollo sostenible, además existe la teoría de los grupos de interés donde los clasifican en accionistas, consumidores, empleados, sociedad y general.

Para Martínez \& Rodríguez (2013); García \& Madero (2016); White \& Alkandari (2019), la óptica de la RSC destaca algunas formas de modelo que se basan exclusivamente a características contextuales; geográficas sociales, culturales, políticas y económicas; es decir, donde se vaya a maniobrar la organización y al sector al que pertenecen. Agregan además que existen modelos que focalizan a la RSC como una herramienta estratégica, políticamente como licencia para instaurarse en el entorno, integradora de las demandas sociales y como requerimientos éticos. Todos estos enfoques conllevan a que las organizaciones dispongan de una imagen ante la sociedad, agregando valor de acuerdo a la manera de comportarse ante las partes implicadas, generándose de esta manera la identidad corporativa.

\section{c) Metodología para la aplicabilidad de la RSC}

La RSC se ha sumergida en cada uno de los eslabones de la organización como símbolo de valor corporativo, para ello es necesario identificar las metodologías utilizadas en el transcurso del tiempo.

\section{- Herramientas de medición de la RSC}

En el mundo empresarial, la RSC se ha transformado en el modelo de comportamiento corporativo a seguir (Alvarado \& Santos, 2019a). Aunque esta nueva tendencia parece imparable, la sociedad todavía mira con bastante escepticismo el nuevo fenómeno de la RSC (Gómez \& Martínez, 2016). No está del todo claro hasta donde las empresas han introducido esa nueva conciencia social en su modelo de negocios, o si simplemente es una nueva campaña de publicidad o marketing verde (Lizcano \& Lombana, 2018). Es decir, si solo es una herramienta que sirve para lavar su cara y vender más a base de convencer al consumidor de que son sostenibles (Acosta, Lovato, \& Buñay, 2018). Algunas empresas han visto en la RSC el camino más corto para conseguir la licencia para operar lo que a menudo se ha traducido en una perversión de la RSC al concebirla como un medio para mejorar la imagen social de la empresa (Muyulema, 2018).

A pesar de que la RSC es voluntaria, dispone de un marco normativo a considerarse para crear herramientas que permitan desarrollar y evaluar la RSC en las instituciones (Burgos, R. 2018). Según Gras, Palacios \& Hernández (2016); Coba, Díaz, Zurita, \& Proaño (2017); Bermúdez, Y., \& Mejías A. (2018), unos de los referentes es el Global Compact, pacto que sirve como 
ISSN: 2602-8506

Vol. 3, $\mathrm{N}^{\circ} 4 .$, p.44-66, octubre - diciembre, 2019

herramienta de autoevaluación; este acoge diez principios básicos de conducta; con el propósito de promover el desarrollo sostenible en las organizaciones y una buena ciudadanía corporativa. Otro de los referentes es la Guía de la Organización para la Cooperación Económica y el Desarrollo- $O C D E$, donde divulgan principios y normas de conductas responsables destinadas para empresas multinacionales. El libro verde de la Unión Europea, es otro referente, donde se detallan líneas de la política gubernamental en esta comunidad de derecho, buscando promover la calidad y la coherencia de las prácticas de responsabilidad social en las organizaciones.

Maigan \& Ferrell (2001), consideró tres procedimientos alternos para examinar el compromiso de las organizaciones con RSC, de diferentes enfoques, siendo estas: evaluación de expertos, indicadores únicos o múltiples, y encuestas a gerentes. Sin embargo, estas herramientas cualitativas fueron ampliadas por Turker (2009), clasificándolas de la siguiente manera: índices de reputación, bases de datos, índices únicos y múltiples, análisis de contenidos de publicaciones, encuestas a miembros de la organización, y escala de medición a nivel individual y organizacional. Según Gangi, Meles, Monferrà, \& Mustilli (2018) estas a su vez se han combinado a fin de obtener información relevante para las diferentes partes implicadas.

De la manera progresiva que se ha venido esclareciendo el direccionamiento de la RSC, se crea un abanico de instrumentos de medición; que, en un inicio, fueron creadas por empresas internacionales para su uso interno a fin de medir el cumplimiento de su único grupo de interés; congregando los procedimientos expuestos por Turker (2009), de acuerdo a características y aspectos contextuales. Entre las herramientas; como modelo de gestión en términos de RSC; más reconocidas y puestos en marcha en las empresas se encuentra Global Reporting Initiative (GRI), establecida en el año 1997 por el Programa Medioambiental de Naciones Unidas y la Coalición de ONG norteamericanas para el desarrollo de economías responsables medioambientales - CERES, esta guía permitió a pequeñas y medianas empresas en todo el mundo adaptar indicadores de cumplimiento a fin de emitir informes de sostenibilidad. Otra guía es la AA1000 establecida por Accountability mediante la aplicación de indicadores, proyectada a inclusión de los grupos de interés al proceso interno de la organización. La Organización Internacional de Estandarización también estableció la normativa ISO 14000; la cual enmarcaba términos ambientales; para posteriormente generar la Norma ISO 26000 que se centraría en la responsabilidad social de las empresas.

En este contexto, se puede indicar la variedad de herramientas que han sido efectuadas por organizaciones que han visto la necesidad y la importancia que implica la RSC en las organizaciones, buscando de una u otra menara cooperar y direccionar de diferentes ámbitos, tomando como referencia una serie de principios y valores identificados en la RSC. 


\section{- Aplicación de RSC en empresas de contexto global}

Las organizaciones hoy en día, consideran imprescindible contar con RSC en cada proceso que efectúan, ya sea tomada como instrumento de estrategia competitiva, de manera política, integradora o por principios éticos (Espinosa et al., 2019). La aplicación de RSC lleva a medir el compromiso o aporte que las empresas han efectuado con clientes, trabajadores, accionistas, ONG, gobierno, sindicatos y demás partes implicadas con la organización (Alvarado \& Santos, 2019b). Bajo este contexto, se muestra el caso de un país árabe de Asia Occidental, donde la RSC es incluyente con las organizaciones de Kuwait; enmarcada por la estructura política y económica del gobierno; quienes integran a las organizaciones y el estado para proyectos de RSC, a fin de promover la imagen del país desde un contexto sociocultural (White \& Alkandari, 2019).

Desde el punto de vista de compromiso ético, se destaca el resultado positivo entre la relación comprador-vendedor al desempeñar un trato justo con sus proveedores, lo que incrementa la confianza de la contraparte, impulsada por los comportamientos éticos de los trabajadores mediante el código de conducta (Son, Lee, Ha, \& Nam, 2019).

Otras de las formas de aplicar RSC en las organizaciones es a través de las redes sociales, lo que genera un impacto positivo a la reputación corporativa, como lo señalan Grover, Kumar, \& Ilavarasan (2019), determinaron que comunicar mediante Twitter las diferentes actividades que se efectúan día a día en la organización por parte de los CEOs, permite mejorar la reputación corporativa de la empresa, siendo una estrategia competitiva. Sin embargo, Vo, Xiao, \& Ho (2019), identifican que las opiniones del boca a boca influyen en las redes sociales (Twitter) positivamente si las aerolíneas disponen de RSC, creándose el efecto contrario en las empresas que no aplican RSC.

Desde un horizonte global los autores Amor, Galindo \& García (2019), consideran que las organizaciones que aplican la RSC en los países, lo efectúan de diferentes niveles y esto depende de su entorno, señalando que las empresas mayormente comprometidas se localizan en países europeos; líderes en RS; los países de América focalizan a la RSC desde la ética, y los países del sureste de Asia poco conocen en cuanto a la temática. Partiendo de este estudio, se identificó a las organizaciones con RSC que son modelos a seguir, entre las empresas europeas que disponen de dicho prestigio; según el Instituto Coordenadas de Gobernanza y Economía Aplicada; se encuentra Inditex, Telefónica, BBVA, Bankia, Endesa, Iberdrola, Gas natural Fenosa, CaixaBank, Banco Santander, Grupo Siro, Mapfre, Ferrovial, Mutua Madrileña, Ree, Enagás, Calidad Pascual, Once y su fundación, Eroski, El corte inglés, Grupo Damm, Telepizza y por último Indra. Pero según datos levantados por el Instituto de Reputación en el año 2017 a nivel global son siete las empresas que lideran la RS a nivel global, estas son: Lego, Microsoft, Google, Walt Disney Company, Grupo BMW, Intel y Robert Bosch. Los resultados localizados, 
aclaran que existen entornos que todavía no se visualizan los RSC implantados en las organizaciones debido a que algunas entidades no aplican balances sociales que demuestren tal gestión para con su grupo de interés.

\section{- Importancia de la RSC}

La envergadura de la RSC en las empresas; sin tener en cuenta su tamaño; se centra en la concientización y compromiso de gestionar los recursos en tres ámbitos: económico, social y medioambiental, denominado también como la triple línea de rendimiento (triple bottom line), que conduce hacia la innovación, por medio adecuada y balanceada gestión empresarial (Muyulema, 2018). El dar cumplimiento a los objetivos de desarrollo sostenible (ODS), de manera indirecta estaría asumiendo el término filantrópica y agregadora de valor en diferentes frentes (Martínez, González, \& Díaz, 2018). Las empresas que optan por incorporar la RSC a la misma, dispondrá de identidad corporativa, sostenibilidad y será considerará parte indispensable dentro de su entorno (Alvarado \& Santos, 2019c).

\section{Sector Económico Popular y Solidario del Ecuador}

\section{a) Antecedentes del SEPS en el Ecuador}

El sistema económico ecuatoriano (SEE), surge durante los años 1831-1839, creado con el fin de canalizar los ahorros dispuestos en el país. Progresivamente se fueron implantando entidades bancarias encasilladas por las políticas del Fondo Monetario Internacional, quienes establecieron diferentes cuerpos legales modificados, logrando colapsar al sistema mediante el incremento de entidades intermediarias, lo que marcó una vicisitud en la economía del país (Pucha et al., 2019). La nueva estructura del SEE fue establecida en la Constitución de la República del Ecuador (2008b), enfatizando la reforma realizada en el ámbito social y solidario dentro del artículo N. ${ }^{\circ} 283$, adhiriendo la forma de integración; desde un panorama económico organizacional; anexadas en cuatro grupos: pública, privada, mixta y popular y solidaria. En el Art. N. ${ }^{\circ} 309$, detalla la conformación dentro del Sistema Financiero Ecuatoriano, sectorizándose en pública, privada y popular y solidaria. Además, expande en el Art. N. ${ }^{\circ} 311$ la composición del SFPS en: Cooperativas de Ahorro y Crédito, entidades asociativas o solidarias, cajas y bancos comunales, y por último cajas de ahorro.

El SEPS; reconocido como economía de mercado (1987), economía social de mercado en el año 1998; forma parte histórica visible en constituciones antepuestas en el país (Ruiz \& Jacome, 2013). Sin embargo, en la Constitución de la República del Ecuador (2008b) es considerada como modelo alternativo de desarrollo proyectada a garantizar la producción y reproducción de las condiciones materiales e inmateriales que posibiliten el buen vivir, mediante una relación dinámica y equilibrada entre sociedad, estado y mercado en armonía con la naturaleza. Al mismo tiempo se establece a la economía popular y solidaria como aquellas organizaciones 
ISSN: 2602-8506

Vol. 3, $\mathrm{N}^{\circ} 4 .$, p.44-66, octubre - diciembre, 2019

socioeconómicas que nacen de las bases de la misma gente; sean estas financieras o no financieras; satisfaciendo las necesidades de las personas, siendo un gran referente del desarrollo local.

Bajo este contexto, los cimientos económicos del estado ecuatoriano han centrado sus esfuerzos en el sector popular y solidario, entrelazando cada una de sus sectores estratégicos hacia el buen vivir, basado en la solidaridad, cooperación y reciprocidad, debido a la participación activa de la población menos favorecidas.

\section{b) Base legal del SEPS}

La base legal en la cual se rige el SEPS empieza en la Constitución de la República del Ecuador (2008a), estableciendo en los artículos 283, 309 y 311, dando paso a la Superintendencia de Economía Popular y Solidaria a supervisar y controlar al sector, mediante la Ley orgánica de Economía Popular y Solidaria y del Sector Financiero Popular y Solidario (LOEPS, 2018); siendo el Comité Interinstitucional y el Consejo Consultivo su rector. Recibiendo además la cooperación del Ministerio Coordinador de Desarrollo Social y Junta de Política y Regulación Monetaria y Financiera con el propósito de regular a las vertientes existentes dentro de la SEPS; sector real y sector financiero, respectivamente; el Instituto Nacional de Economía Popular y Solidaria concede asistencia técnica, mientras que la Corporación Nacional de Finanzas Populares y Solidarias otorga financiamiento, tal como se muestra en la Figura 1.

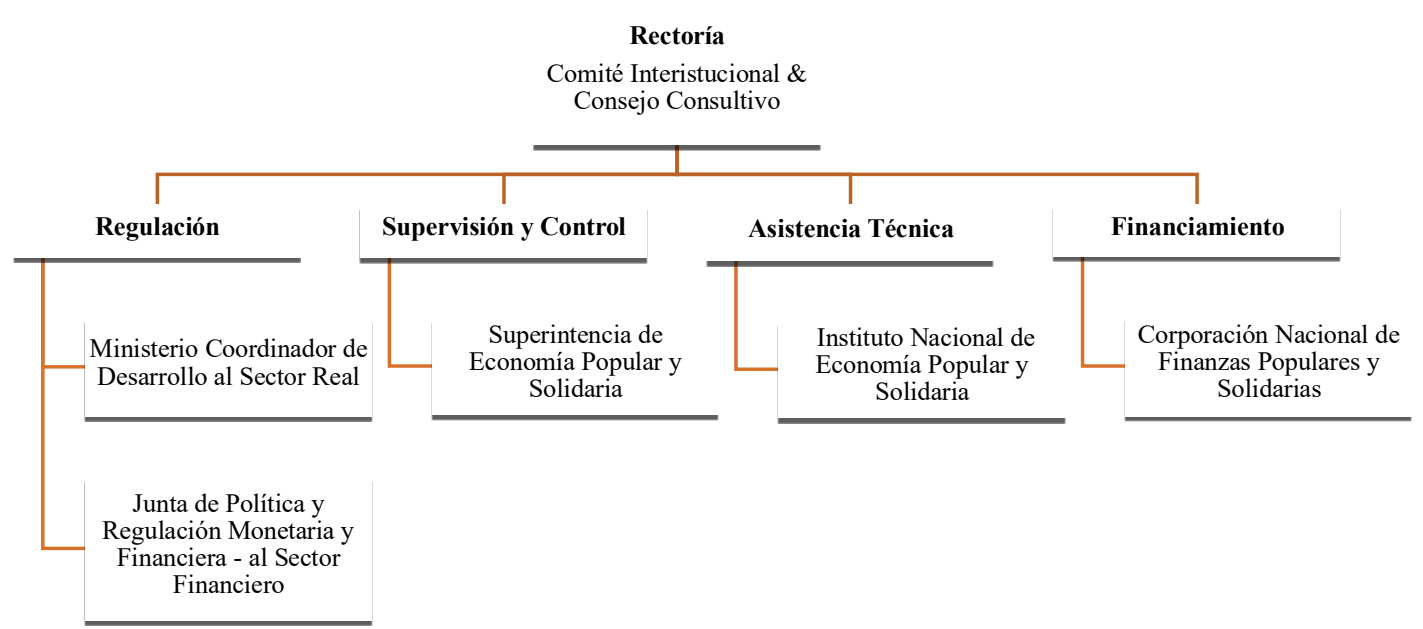

Figura 1. Esquema del Marco Institucional.

Fuente: Elaboración propia a partir de datos publicados por la LOEPS (2018).

Estas instituciones trabajan conjuntamente a fin de armonizar e ir encaminadas a los principios y valores establecidos en la constitución, permitiendo de esta manera incluir a la ciudadanía en la participación activa de la economía del país siendo su principal objetivo de otorgar un trato especial y digno que viabilice su desarrollo como individuo. 
ISSN: 2602-8506

Vol. 3, N4., p.44-66, octubre - diciembre, 2019

\section{c) Estructura del SEPS y su evolución}

El SEPS en el Ecuador cuenta con normas y entidades de control específicas y diferenciadas, encargadas de preservar su seguridad, estabilidad, transparencia y solidez. Este sector, en un principio, se hallaba controladas por la Superintendencia de Bancos y Seguros-SBS y Dirección Nacional de Cooperativas-DNC, las mismas que trasladaron; en el 2013 y 2012, respectivamente; la supervisión y control a la Superintendencia de Economía Popular y Solidaria, misma que dispone de normativa e institucionalidad específica para el sector (Pucha et al., 2019).

La SEPS, supervisada y controlada por la Superintendencia de EPS, inicialmente trabajó en conjunto con SBS y DNC para conocer la naturaleza, estructura, gestiones efectuadas y la dimensión de las entidades que pertenecen del mismo, a fin de disponer de información subsanada y que sirva de línea base. Esta edificación de la información presentó diferentes anomalías; registros duplicados e inexistentes, clasificaciones erróneas, inconsistencias en información financiera; que de manera progresiva se fue saneando y dictaminando jurídicamente, permitiendo mejorar su gobernabilidad, su compromiso con la colectividad, organizar de manera más eficiente sus procesos de control, sus esquemas financiero, su gestión administrativa y transparencia; de acuerdo a las características, valores y principios por la cual se caracteriza el sector (Ruiz \& Jacome, 2013). El SEPS, fue identificando particularidades en las organizaciones que formaban parte del sector, que en un inicio no se concebían, tal como se muestra en la Figura 2.

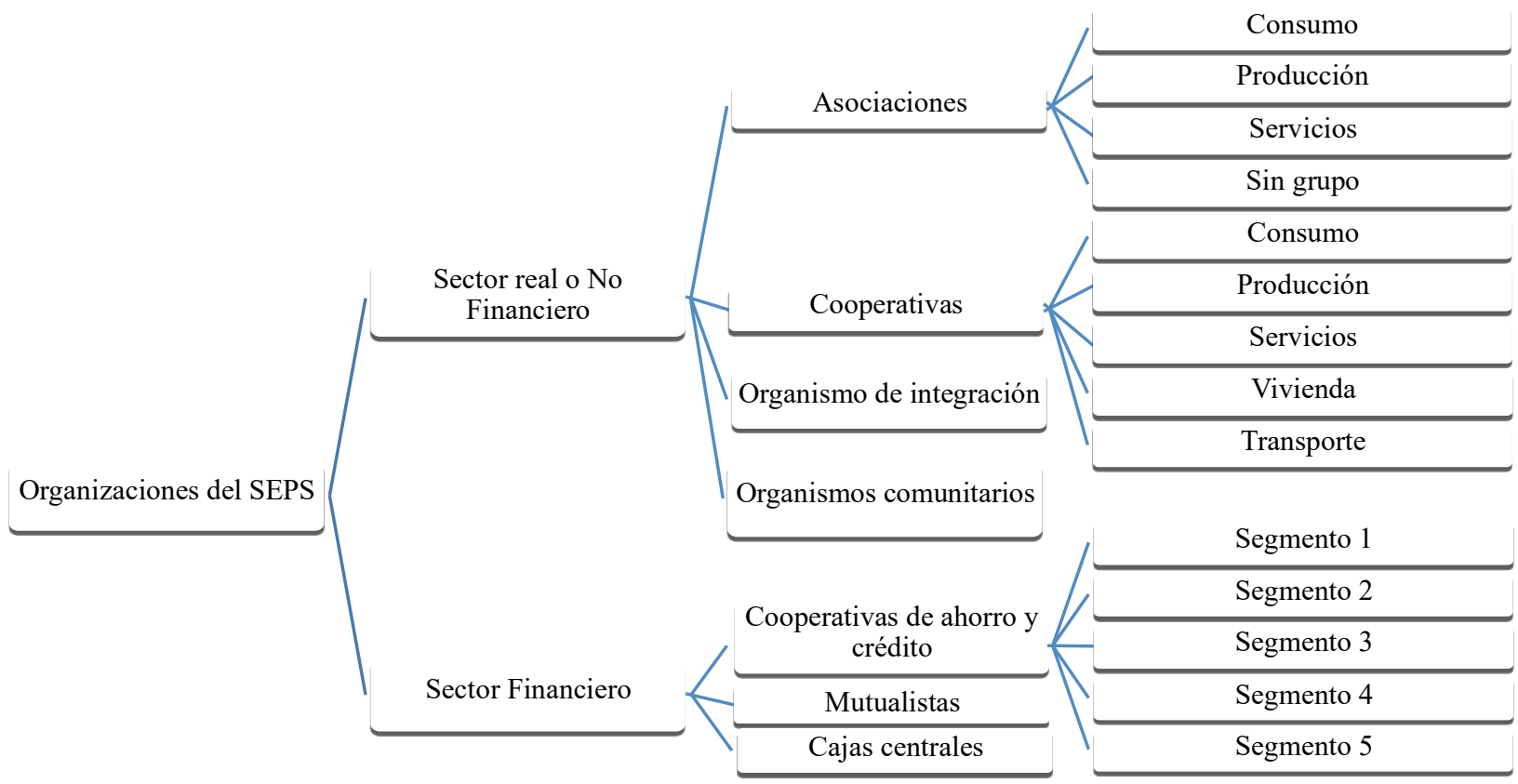

Figura 2. Estructura del Sector Económico Popular y Solidario.

Fuente: Elaboración propia a partir de datos publicados por la Superintendencia de EPS \& LOEPS (2018). 
ISSN: 2602-8506

Vol. 3, N4., p.44-66, octubre - diciembre, 2019

La clasificación en la Figura 2, permitió agrupar organizaciones del sector real o no financiero y el sector financiero. Este primero se encuentra compuesto por asociaciones, cooperativas, organismos de integración y organismos comunitarios. Al segundo grupo del SEPS, se le atribuyen las cooperativas de ahorro y crédito, mutualistas y cajas centrales, que han sido segmentadas; de manera progresiva; en cinco grupos de acuerdo al monto de sus activos.

De conformidad al catastro dispuestos en la Superintendencia de Economía Popular y Solidaria (2019), el Sector No Financiero dispone de 16082 organizaciones, de las cuales una se encuentra en intervención, 205 en proceso de liquidación, 274 se encuentran liquidadas, 1163 inactivas y 14439 activas, representando el 90\% de total registradas. Este último grupo de empresas, 7336 de estas pertenecen al fragmento de producción, 6776 son organizaciones de servicio, $117 \mathrm{se}$ encuentran conformado por grupo de servicio, 173 agrupaciones se identificaron por grupo de vivienda, mientras que 35 no disponen clasificación. Del informe se puede destacar adicionalmente que, del total de organizaciones registradas en el sector no financiero, el $10 \%$ (1643) no se encuentran efectuando su labor, acaparando el 7\% de este grupo en organizaciones de producción, seguidamente del grupo de servicio y vivienda, con el $2 \%$ con similar porcentaje, tal como se exterioriza en la Figura 3.

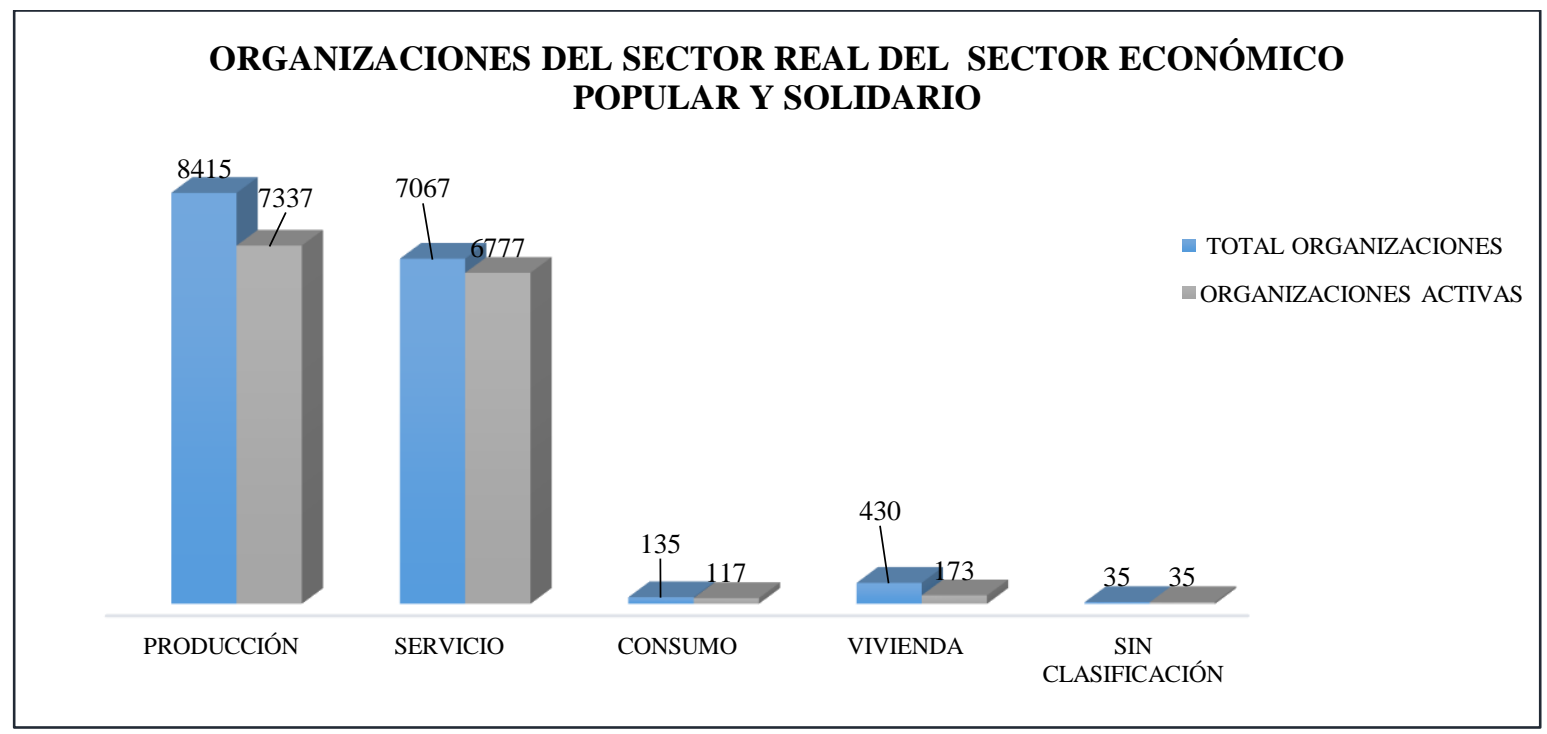

Figura 3. Organizaciones del Sector No Financiero vs las activas dentro del sector de acuerdo al grupo o segmento. Fuente: Elaboración propia a partir de datos publicados por la SEPS (2019).

Según el reporte otorgado por la Superintendencia de Economía Popular y Solidaria (2019), 576 entidades forman parte del sector financiero. Donde 34 Cooperativas de Ahorro y Crédito (Coac) se encuentran segmentadas en el grupo 1 y 4 mutualistas, en el segmento 2 se localizan Coac 42, 83 Coac en el segmento 3, 169 en el segmento 4, y 244 en el segmento 5, tal como se expone en la Figura 4. 


\section{SEGMENTACIÓN DE LAS ORGANIZACIONES DEL SECTOR FINANCIERO POPULAR Y SOLIDARIO -2019}

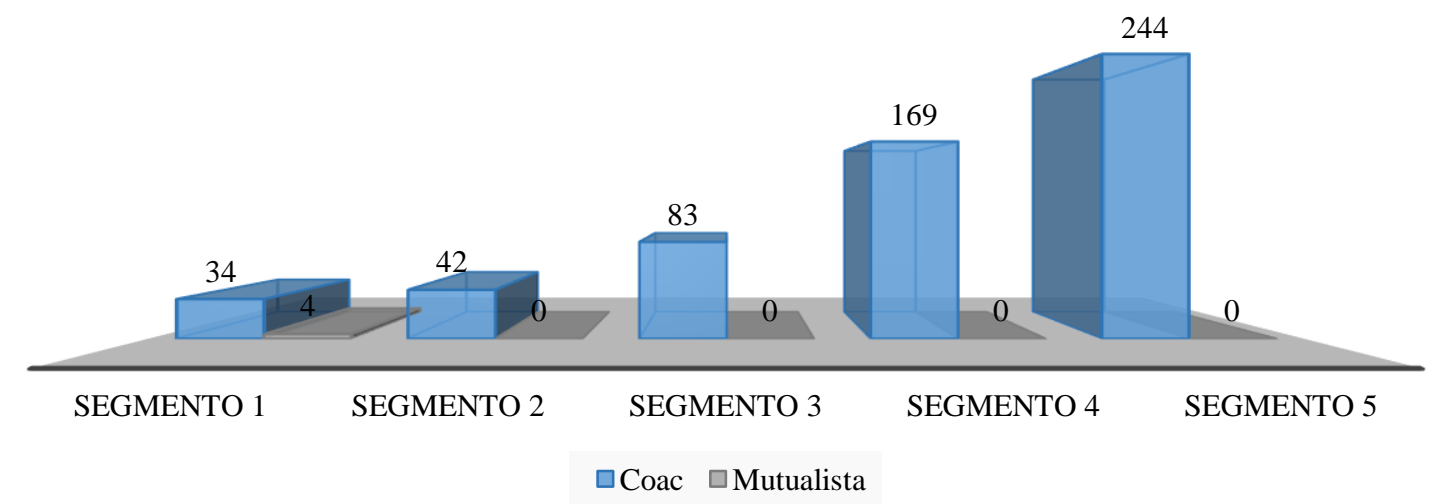

Figura 4. Organizaciones del Sector Financiero Popular y Solidario.

Fuente: Elaboración propia a partir de datos publicados por la SEPS (2019).

A pesar de contar con mayores organizaciones en el Sector No Financiero dentro de la EPS, no se visualiza por parte de la Superintendencia los mecanismos e información relevante de esta parte de la economía del país, a fin de incluir a los ciudadanos a través de incentivos para un correcto funcionamiento y mantenerse a través del tiempo, a pesar de contar con estrategias y políticas ya establecidas para el sector. Esto ha hecho que algunas organizaciones del sector real cierren y paralicen sus actividades, provocando desconfianza de las entidades del sector público encargadas del sector, aplazando los nuevos emprendimientos por falta de asistencia e iniciativas en las estrategias planteadas, que conlleven a reactivar de manera dinámica e inclusiva al sector real.

\section{Discusión}

\section{Los retos actuales del SEPS frente a las RSC}

\section{a) Relación entre la RSC y SEPS ecuatoriano}

En Ecuador se ha efectuado un plan estratégico donde se enmarcan procesos, políticas y lineamientos asumiendo la visión ancestral de los pueblos indígenas; fundamentados en la solidaridad, respeto, armonía y equilibrio con la naturaleza; como alternativa política y con la finalidad de la búsqueda del Sumak Kawsay (Buen Vivir), sirviendo el SEPS como instrumento para cumplir con el plan estratégico a nivel nacional.

Las dimensiones de la Responsabilidad Social Corporativa, guarda mucha analogía con los principios del SEPS. De los ocho principios establecidos en el artículo 4 de la LOEPS (2011) para el SEPS, cinco incluyen las bases de comportamiento de RSC, 1) la búsqueda del buen vivir y del bien común, 2) la prelación del trabajo sobre el capital y de los intereses colectivos 
ISSN: 2602-8506

Vol. 3, N4., p.44-66, octubre - diciembre, 2019

sobre los individuales, 3) el comercio justo y consumo ético y responsable, 4) la equidad de género, y 5) la responsabilidad social y ambiental, la solidaridad y rendición de cuentas. Al igual que los principios, los valores del sector fueron direccionados al desarrollo sostenible, mostrando al ser humano como principio y fin, asumiendo en ellos justicia, honestidad, transparencia y responsabilidad social, por medio de la cooperación, esfuerzo propio, gestión democrática, comercio justo y consumo ético.

Tabla 2. Relación entre las dimensiones de la RSC y los principios de la SEPS establecidas en la LOEPS

\begin{tabular}{|c|c|c|}
\hline $\begin{array}{l}\text { Dimensiones de la } \\
\text { RSC - Desarrollo } \\
\text { sostenible }\end{array}$ & $\begin{array}{l}\text { Principios de SEPS } \\
\text { según la LOEPS }\end{array}$ & Descripción \\
\hline Económico & $\begin{array}{l}\text { a) La búsqueda del buen } \\
\text { vivir y del bien común; }\end{array}$ & $\begin{array}{l}\text { Se centra en el apoyo o cooperación de los individuos, a fin } \\
\text { de solucionar necesidades, a través de la generación de } \\
\text { condiciones, donde el bienestar del ser humano es la } \\
\text { prioridad. }\end{array}$ \\
\hline Medioambiental & $\begin{array}{l}\text { b) La prelación del } \\
\text { trabajo sobre el capital y } \\
\text { de los intereses } \\
\text { colectivos sobre los } \\
\text { individuales; }\end{array}$ & $\begin{array}{l}\text { Hace énfasis sobre la visión compartida, donde las personas } \\
\text { proyectan laborar de manera conjunta y organizada para } \\
\text { cubrir sus necesidades; utilizando al capital como un } \\
\text { conducto; a través de la distribución equitativa de los } \\
\text { ingresos generados. }\end{array}$ \\
\hline \multirow[t]{3}{*}{ Social } & $\begin{array}{l}\text { c) El comercio justo y } \\
\text { consumo ético y } \\
\text { responsable: }\end{array}$ & $\begin{array}{l}\text { Se trata de proveerse de la naturaleza de manera } \\
\text { comprometida, sin afectar el bienestar del entorno ni de los } \\
\text { demás individuos que en el habitan. }\end{array}$ \\
\hline & d) La equidad de género; & $\begin{array}{l}\text { Condiciones iguales en participar y contribuir de manera } \\
\text { justa en los diferentes ámbitos y entornos de la sociedad. }\end{array}$ \\
\hline & $\begin{array}{l}\text { e) La responsabilidad } \\
\text { social y ambiental, la } \\
\text { solidaridad y rendición } \\
\text { de cuentas. }\end{array}$ & $\begin{array}{l}\text { Unifica la producción y reproducción para satisfacer de } \\
\text { mejor manera las necesidades concertadas por la misma } \\
\text { colectividad, respetando la naturaleza al tomar lo necesario } \\
\text { a fin de que estos recursos perduren para las futuras } \\
\text { generaciones. Al mismo tiempo apoyarse mutuamente y que } \\
\text { estas actividades sean visibilizadas por los integrantes con el } \\
\text { propósito de verificar que se está actuando de manera } \\
\text { correcta sin afectar el entorno al cual son parte activa. }\end{array}$ \\
\hline
\end{tabular}

Fuente: Los autores basados en LOEPS (2011); Martínez \& Rodríguez (2013); Schoenmaker (2017); Pucha et al., (2019).

La magnitud de semejanza entre estas dos terminologías, han permitido identificar la cooperación que incurren el SEPS en la economía para el bienestar del ser humano; logrando una cercanía y sensación de proximidad con los individuos que integra elementos de dignidad regional y un sorprendente grado de adaptación a los cambios habidos en el entorno y el comportamiento del ser humano, por lo que se puede asegurar que en el sector popular y solidario ya se ha venido practicando la RSC desde hace muchos decenios, bajo el epígrafe del cooperativismo. 
ISSN: 2602-8506

Vol. 3, N4., p.44-66, octubre - diciembre, 2019

\section{b) Barreras existentes en el SEPS en la inserción de la RSC}

Entre las barreras existentes dentro de la Superintendencia para el SEPS, está la normativa (LOEPS) y su Reglamento a la Ley Orgánica de la Economía Popular y Solidaria y del Sector Financiero Popular y Solidaria (RLOEPS), donde no se contempla ninguna herramienta, proposición sustantiva o guía práctica que permita acreditar de manera fidedigna los procesos de responsabilidad social por parte de las organizaciones del sector.

Cabe señalar que, durante el año 2014, se levantó información sobre la RSC a través de un balance social de las cooperativas del SEPS, a fin de medir la gestión; en términos de la misión, visión mediante los principios y valores establecidos en la LOEPS; efectuada durante los años 2012 y 2013; mediante una encuesta enviada a las cooperativas que forman parte del SEPS; como prueba piloto. Con información obtenida del 39\% solicitada; proveniente del 61\% del sector real y el 39\% del sector financiero; sobre los años 2012 y 2013 (Páez, 2014).

Entre los resultados encontrados en el balance social de las cooperativas que integran el SEPS, el 56\% son hombre y el $44 \%$, pero las mujeres superan su participación en el sector financiero a diferencia del sector no financiero, que esta mayormente compuesto por hombres socios de estas cooperativas. El $46 \%$ de las cooperativas no dispone de política salarial. En términos de primacía de los intereses colectivos sobre los individuales, el $61 \%$ de las organizaciones manifestó no haber tratado sobre el tema. El $34 \%$ establece que existen documentos con los procedimientos a efectuar la incorporación de socios y en cuanto a su salida, sostiene el $38 \%$ la existencia de una política clara y socializada. En cuanto a la composición de los órganos de dirección en estas organizaciones de acuerdo al género, existe un promedio del $75 \%$ de los puestos es ocupado por hombres. Otro dato relevante obtenido de esta información, es que a pesar que existen un $52.62 \%$ lo que encierra las palabras "gobierno cooperativo", apenas el $22.96 \%$ afirmó disponer de dicho código en su entidad y el 33\% poseer código ético. El $41.9 \%$ dispone de medidas de lucha contra la corrupción. Sin embargo, hay temas en cuanto a la responsabilidad social que no se han tratado en las cooperativas, como es el caso de educación, capacitación y comunicación - Inter cooperación e integración sectorial - compromiso social, solidario, comunitario y ambiental para su personal y socios.

Estos resultados permitieron conocer la identidad de la organización para; con base a la primera información obtenida; mejorar el cumplimiento del sector, en pos de generar responsabilidad social y solidaria, concibiendo confianza entre los participantes y la ciudadanía en general.

Hoy, existe información financiera actualizada de una parte del SEPS; el sector financiero; imposibilitando conocer la gestión efectuada en materia de responsabilidad social y solidaria; y la evolución en la misma; del sector real y sector financiero. En la actualidad la Superintendencia de EPS ha tratado de que las entidades bajo su supervisión presenten un balance social, pero no existe normativa para tal solicitud ni formatos de los parámetros requeridos en dicho documento que sirva de modelo dispuesto en la página oficial de dicha entidad. 


\section{c) Retos de la RSC frente a la SEPS}

Entre los retos que enfrenta el SEPS se encuentra en estructurar una herramienta clara y sencilla a fin de verificar el cumplimiento de los principios y valores cooperativistas encaminados a la misión y visión para medir la gestión desde un enfoque de triple criterio: económico, social y medioambiental.

Posteriormente socializar y capacitar a cada una de las organizaciones que forman parte del SEPS, poniendo a disposición del sector la herramienta y asistencia técnica para el buen manejo de la misma, sirviendo de guía para los demás sectores.

El crear un balance social y solidario enraizando a la RSC y los principios del SEPS permitirá acentuar su identidad y alentar la confianza del sector como de la ciudadanía, a más de integrar a todos los sectores del mercado a través de la participación activa y democrática de sus integrantes, llegando a crear organizaciones humanizadas que respetan su entorno.

\section{Conclusiones}

A pesar de la no existencia de una definición unánime sobre Responsabilidad Social Corporativa (RSC), existen entidades que le dan peso a este término y la importancia de este, incorporarlo de forma voluntaria en las organizaciones, que buscan mantenerse en el tiempo y ser parte activa del entorno donde habitan. La disponibilidad de herramientas aplicadas globalmente en las organizaciones, ha hecho visible el triple aporte de rendimiento; en términos económico, social y ambiental; efectuado por la RSC, concibiendo gestión, confianza, respaldo de la sociedad y valor corporativo, mediante la transparencia y coherencia.

El Sector Económico Popular y Solidario (SEPS) es uno de los pilares del sistema económico del Ecuador; incluida en la Constitución de la República del Ecuador (2008b); acogiendo a las organizaciones económica - social, que buscan satisfacer necesidades y generar ingresos a todos sus integrantes; considerado como factor de dinamización y crecimiento económico, fuente de capacidades empresariales y de empleos productivos. Actualmente existen 15015 organizaciones activas; 14439 forman parte del sector no-financiero y 576 del sector financiero; las mismas que disponen de subclasificación de acuerdo a características y patrones identificadas a fin de generar políticas y estrategias específicas.

La analogía entre las dimensiones de RSC con los principios del SEPS es visible como parte innata de su concepción. Las organizaciones del SEPS; por su naturaleza; han efectuado actividades de forma responsable a fin de guiar su accionar, las mismas que no han sido plasmadas para dar a conocer a su grupo de interés. El aporte de la RSC hacia el SEPS, es plasmar memorias de sostenibilidad y desarrollando sistemas de indicadores económicos, sociales y medioambientales en su gestión para mostrar su comportamiento que permita reafirmar su identidad.

En este sentido, las ventajas de implantar la RSC a las organizaciones del SEPS son considerables para la reactivación económica por sus aportaciones en el progreso del conocimiento, combinando los procedimientos y valores propios de la EPS, al incorporar a ello la base de la triple línea de rendimiento con las de los sectores emergentes, coadyuvando a la seguridad para el buen vivir. 


\section{Referencias bibliográficas}

Acosta, V. M., Lovato, T. S., \& Buñay, C. J. (2018). La responsabilidad social corporativa y su rol en las empresas ecuatorianas. Revista Lasallista de Investigación, 15(2), 105-117. doi:10.22507/rli.v15n2a8

Alvarado, M. S., \& Santos, T. M. (2019). La responsabilidad social como estrategia de protección social en el capitalismo contemporáneo. Revista Katálysis, 22(1), 142-150. doi:10.1590/1982-02592019v22n1p142

Amor, V., Galindo, M., \& García, I. (2019). A Multivariate Proposal for a National Corporate Social Responsibility Practices Index (NCSRPI) for International Settings. Social Indicators Research, 36 (525-560).

Arteaga Macías, R. S. (2017). Economía popular y solidaria, República del Ecuador. Dominio de las Ciencias, 5(3), 533-542. doi: http://dx.doi.org/10.23857/dom.cien.pocaip.2017.3.4.oct.533-542

Bermúdez Colina, Y., \& Mejías Acosta, A. A. (2018). Medición de la responsabilidad social empresarial: casos en pequeñas empresas Latinoamericanas. Ingeniería Industrial, XXXIX (3), 315-325.

Burgos, R. C. (2018). La Responsabilidad Social o sostenibilidad: un enfoque desde el entorno y la comunicación. RETOS. Revista de Ciencias de la Administración y Economía, 8(16), 1-16. doi: https://doi.org/10.17163/ret.n16.2018.04

Carro Suáreza, J., Sarmiento Paredes, S., \& Rosano Ortega, G. (2017). La cultura organizacional y su influencia en la sustentabilidad empresarial. La importancia de la cultura en la sustentabilidad empresarial. Estudios Gerenciales, 33, 352-365. doi: https://doi.org/10.1016/j.estger.2017.11.006

Chávez, R., \& Monzón, J. (2018). La economía social ante los paradigmas económicos emergentes: innovación social, economía colaborativa, economía circular, responsabilidad social empresarial, economía del bien común, empresa social y economía solidaria. CIRIEC-España. Revista de Economía Pública, Social y Cooperativa, 93, 5-50. doi: https://doi.org/10.7203/CIRIEC-E.93.12901

Coba, M. E., Díaz, C. J., Zurita, M. E., \& Proaño, L. P. (2017). La responsabilidad social empresarial en las empresas del Ecuador. Un test de relación con la imagen corporativa y desempeño financiero. Ingeniería Industrial. Actualidad y Nuevas Tendencias, V(18), 23-44. 
Vol. 3, N4., p.44-66, octubre - diciembre, 2019

Constitución de la República del Ecuador. (2008a). Capítulo Cuarto-Soberanía económica. Sección primera: Sistema económico y política económica. Art. 283. Montecristi: Asamblea Nacional Constituyente de Ecuador.

Constitución de la República del Ecuador. (2008b). Capítulo Cuarto-Soberanía económica. Sección Octava: Sistema Financiero. Art. 309. Montecristi: Asamblea Nacional Constituyente de Ecuador.

Espinosa Ruiz, C. G., Muyulema Allaica, J. C., Sánchez Macías, R. A., \& Usca Veloz, R. B. (2019). Los retos sostenibilistas de las Pymes textileras de la provincia de Tungurahua - Ecuador. RIIIT. Revista Internacional de Investigación e Innovación Tecnológica, 7(38), 1-19.

Gangi, F., Meles, A., Monferrà, S., \& Mustilli, M. (2018). Does corporate social responsibility help the survivorship of SMEs and large firms? Global Finance Journal. doi: https://doi.org/10.1016/j.gfj.2018.01.006

García, A., Gómez, O., Mera, Ó. A., \& Pabón, F. (2014). Evolución de la responsabilidad social empresarial y la experiencia en el sector financiero. Revista TEMAS, 2(8), 249-260.

García Santos, J. J., \& Madero Gómez, S. (2016). La Evolución del Concepto de Responsabilidad Social Corporativa: Revisión litera. Conciencia Tecnológica, 51, 3846.

Gómez, N. B., \& Martínez, D. R. (2016). Los valores éticos en la responsabilidad social corporativa. Anagramas, 14(28), 33-50 .

Gras Gil, E., Palacios, M. M., \& Hernández, F. J. (2016). Investigating the relationship between corporate social responsibility and earnings management: Evidence from Spain. $B R Q$ Business Research Quarterly, 19(4), 289-299. doi: https://doi.org/10.1016/j.brq.2016.02.002

Grover, P., Kumar, K. A., \& Ilavarasan, P. V. (2019). Impact of corporate social responsibility on reputation-Insights from tweets on sustainable development goals by CEOSs. International Journal of Information Management, 14 (39-52).

Horváthová, E. (2010). Does environmental performance affect financial performance? A metaanalysis. Ecological $\quad$ Economics, $\quad 70(1), \quad 52-59 . \quad$ doi: https://doi.org/10.1016/j.ecolecon.2010.04.004

Lizcano Prada, J., \& Lombana, J. (2018). Responsabilidad Social Corporativa (RSC): reconsiderando conceptos y enfoques 1. Civilizar Ciencias Sociales y Humanas, 18(34), 119-134. doi:10.22518/usergioa/jour/ccsh/2018.1/a08 
Vol. 3, N4., p.44-66, octubre - diciembre, 2019

LOEPS (Ley Orgánica de Economía Popular y Solidaria y del Sector Financiero Popular y Solidario). (2018). Reforma al Registro Oficial 444. Sección 3.- De las Entidades Asociativas o Solidarías, Cajas y Bancos Comunales y Cajas de Ahorro. Quito: Asamblea Nacional Constituyente de Ecuador. Obtenido de http://www.seps.gob.ec/documents/20181/25522/LEY\%20ORGANICA\%20DE\%20E CONOMIA\%20POPULAR\%20Y\%20SOLIDARIA\%20actualizada\%20noviembre\%2 02018.pdf/66b23eef-8b87-4e3a-b0ba-194c2017e69a

LOEPS, L. O. (2011). Capítulo primero - Marco conceptual y clasificación. Quito: Asamblea Nacional Constituyente de Ecuador.

Maigan, I., \& Ferrell, O. (2001). Measuring corporate citizenship in two conuntries: The case of the United State and France. Journal of Business Ethics, 23 (283 - 297).

Martínez, P., \& Rodríguez del Bosque, I. (2013). Revisión teórica del concepto y estrategias de medición de la responsabilidad social corporativa. Prisma Social, 11, 321-350.

Muyulema Allaica, J. C. (2018). La ecología industrial y la economía circular. Retos actuales al desarrollo de industrias básicas en el Ecuador. Dilemas Contemporáneos: Educación, Política y Valores, 5(2)., 1-15.

Orlitzky, M., \& Shen, J. (2013). Corporate Social Responsibility, Industry, and Strategy. Industrial and Organizational Psychology, 6(4), 346-350. doi:https://doi.org/10.1111/iops.12064

Páez, P. J. (2014). Balance social. Modelo y primeros resultados agregados. Quito: III Jornadas de supervisión y control. Función de Transparencia y Control Social FTCS. Obtenido de https://www.seps.gob.ec/documents/20181/26626/BS\%20Modelos\%20y\%20primeros \%20resultados\%20agregados_Jose\%20Ramon\%20Paez\%20Pareja_III\%20Jornadas.pd f/dd279fab-ff29-4a84-9614-31eff188e68f

Pucha, M. P., Muyulema Allaica, J. C., Burgos, A. C., \& Buenaño, B. E. (2019). Gestión de la calidad como estructura del desempeño operacional en el sector Cooperativo Financiero del segmento cinco de la provincia de Chimborazo. Revista Dilemas Contemporáneos: Educación, Política y Valores (2(78)), 1-28.

Ruiz, R. M., \& Jacome, H. (2013). El sector económico popular y solidario en Ecuador: Diagnóstico y modelo de supervisión. Estudios sobre economía popular y solidaria, 101-144. 
Vol. 3, N4., p.44-66, octubre - diciembre, 2019

Sahut, J. M., Peris Ortiz, M., \& Teulon, F. (2019). Corporate social responsibility and governance. Journal of Management and Governance, 1-12.

doi:https://doi.org/10.1007/s10997-019-09488-8

Saltos, C. J., Mayorga, D. M., \& Ruso, A. F. (2016). La economía popular y solidaria: un estudio exploratorio del sistema en Ecuador con enfoque de control y fiscalización. Cofin Habana, 11(2), 55-75.

Schoenmaker, D. (2017). From Risk to Opportunity: A Framework for Sustainable Finance. Países Bajos: Rotterdam School of Management, Eramus University.

Son, B.G., Lee, C.H., Ha, B.C., \& Nam, H. (2019). Investigating the fair treatment of suppliers and its trust fostering role and performance benefits. International Journal of Production Economics, 13 (54-66).

Superintendencia de Economía Popular y Solidaria (SEPS). (1 de agosto de 2019). Noticias de la Superintendencia de Economía Popular y Solidaria. Obtenido de Noticias de la Superintendencia de Economía Popular y Solidaria: https://www.seps.gob.ec/noticia?segmentacion-de-entidades-del-sector-financieropopular-y-solidario-ano-2019

Torres, G. J., \& Cano, M. A. (2019). Importancia del Gobierno Corporativo en las sociedades financieras populares en México. Cuadernos de Contabilidad, 20(49), 01-16. doi:https://doi.org/10.11144/Javeriana.cc20-49.igcs

Turker, D. (2009). Measuring corporate social responsibility: A scale development study. Journal of business ethics, 85.

Vega, U. S. (2016). La política de Economía Popular y Solidaria en Ecuador. Una visión de su gubernamentalidad. Otra Economía, 10(18), 77-90. doi: 10.4013/otra.2016.1018.07

Vo, T. T., Xiao, X., \& Ho, S. Y. (2019). How Does Corporate Social Responsibility Engagement Influence Word of Mouth on Twitter? Evidence from the Airline Industry. J Bus Ethics, 18 (535-543).

White, C., \& Alkandari, K. (2019). The influence of culture and infrastructure on CSR and country image: The case of Kuwait. Public Relations Review, 45(3), 101783. doi:https://doi.org/10.1016/j.pubrev.2019.05.004 
ISSN: 2602-8506

Vol. 3, N4., p.44-66, octubre - diciembre, 2019

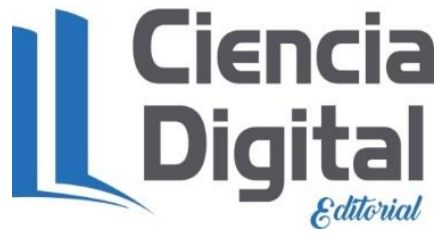

\section{PARA CITAR EL ARTÍCULO INDEXADO}

Muyulema Allaica, J., Pucha Medina, P., Villamarín Arévalo, A., \& Muyulema Allaica, C. (2019). Desafíos de la responsabilidad social corporativa (RSC) en el sector económico popular y solidario del Ecuador. Visionario Digital, 3(4), 44-66.

https://doi.org/10.33262/visionariodigital.v3i4.965

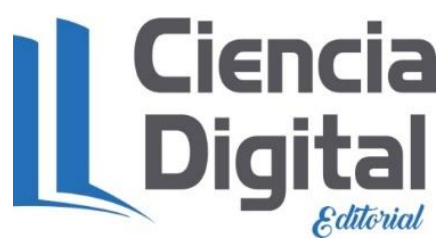

El artículo que se publica es de exclusiva responsabilidad de los autores y no necesariamente reflejan el pensamiento de la Revista Visionario Digital.

El artículo queda en propiedad de la revista y, por tanto, su publicación parcial y/o total en otro medio tiene que ser autorizado por el director de la Revista Visionario Digital.
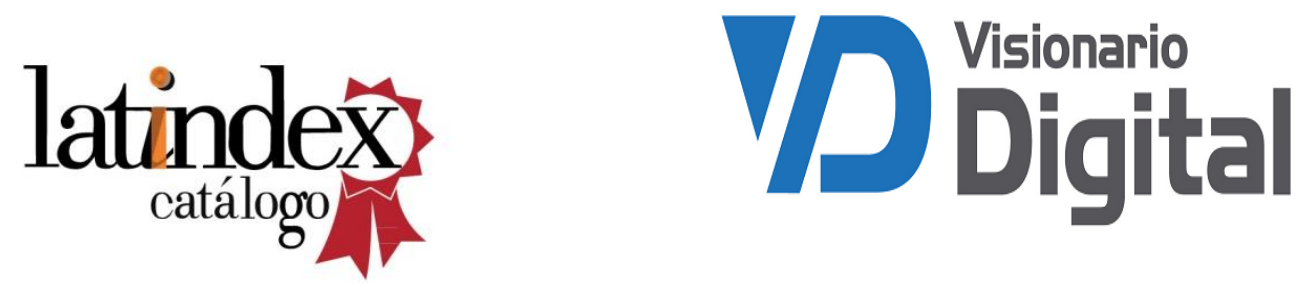